\title{
The Glarus overthrust : a major pathway for the escape of fluids out of the Alpine orogen
}

\author{
Nicolas P. Badertscher \\ Institut de Géologie, Université de Neuchâtel, Emile-Argand 11, 2007 Neuchâtel, Switzerland \\ Georges Beaudoin \\ René Therrien \\ MEDEF, Département de Géologie et de Génie Géologique, Université Laval, Québec G1K7P4, \\ Canada \\ Martin Burkhard* \\ Institut de Géologie, Université de Neuchâtel, Emile-Argand 11, 2007 Neuchâtel, Switzerland
}

*Corresponding author: Martin.burkhard@unine.ch.

${ }^{1}$ GSA Data Repository item 2002105, is available from Documents Secretary, GSA, P.O. Box 9140, Boulder, CO 80301-9140, editing@geosociety.org, or at www.geosociety.org/pubs/ft2002.htm.

\section{DATA Repository :}

As complement to Figure 2 of the original article, 12 additional fluid modelling configurations, out of a modelling study with more than 100 runs are illustrated. These models carry their original numbers :

S23, S24, S25, S32, S34, S38, S66, S72, S76, S77, S78, S81

For each model, physical properties of the different lithologies are listed in a summary table (top left). The geometric configuration of source(s) and sink(s) are illustrated in a block diagram (top right). Resulting hydraulic heads as well delta-18-O patterns after $25^{\prime} 000,100^{\prime} 000$ and 500'000 years modelling time respectively are illustrated in color coded block-diagrams. 


\section{Fignre 2 (of article)}

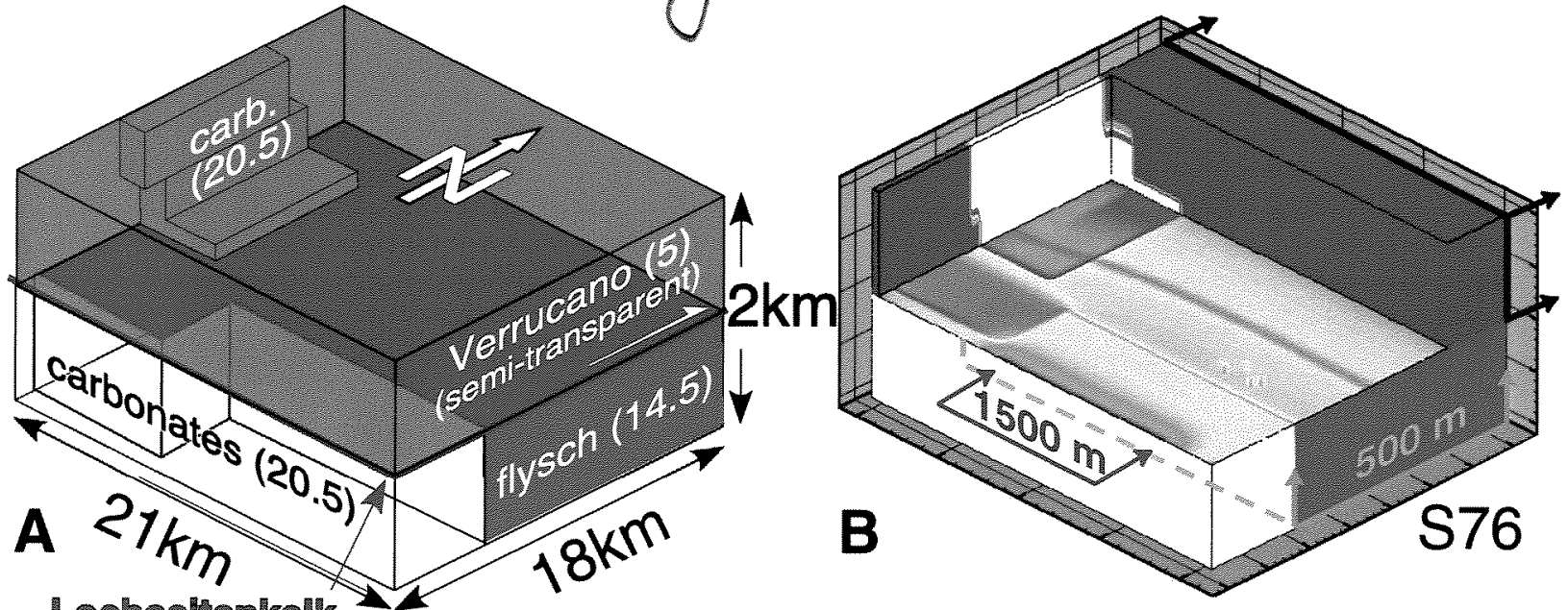

\section{Lochseltenkalk calcite $\delta^{180}$ (\% VSMOW)}
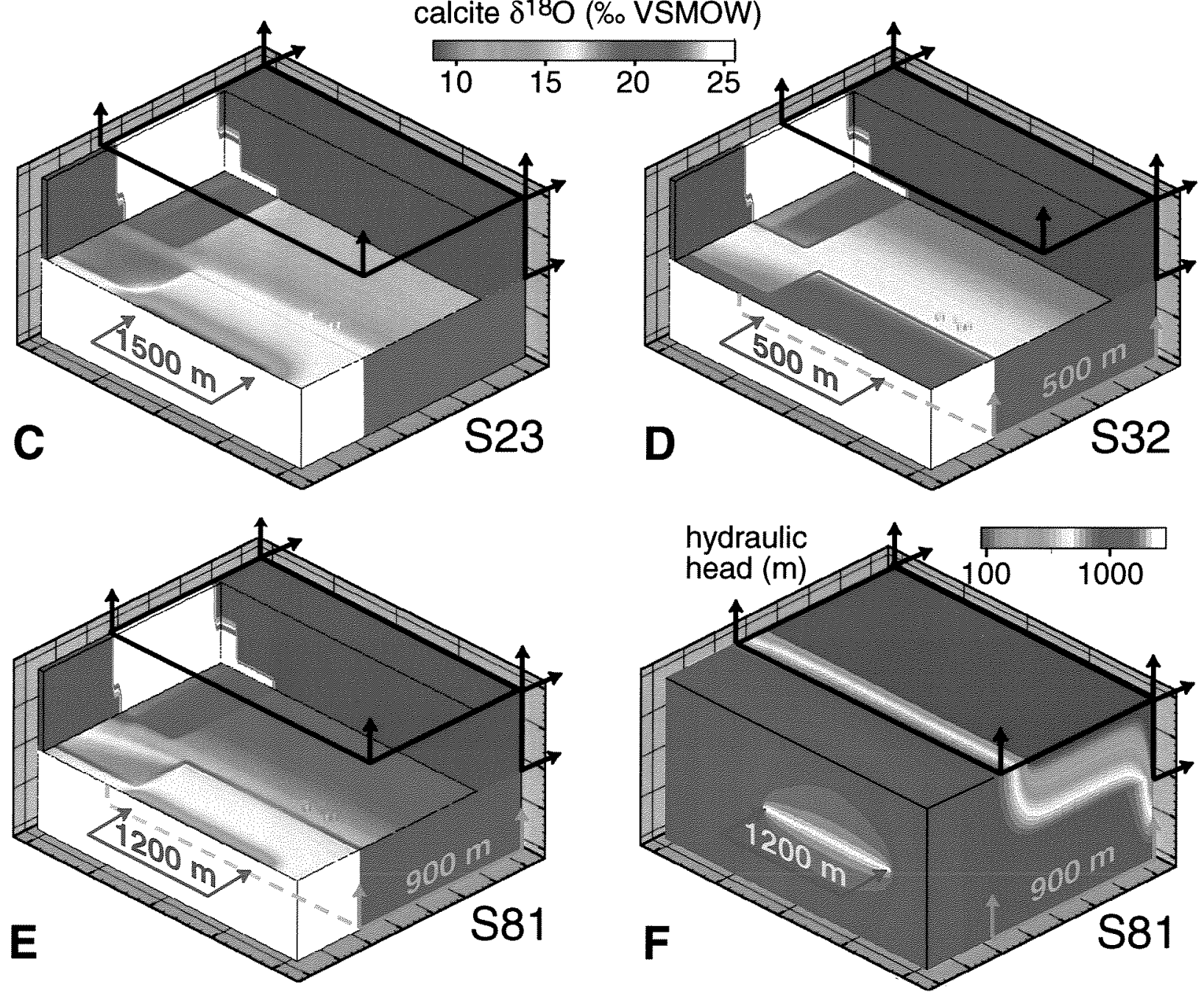
S23

Basement head: 1500

\begin{tabular}{|l|l|l|l|l|l|}
\hline Lithology & $\mathrm{Kxx}$ & $\mathrm{Kyy}$ & $\mathrm{Kzz}$ & $\emptyset$ & $\mathrm{Xkk}$ \\
\hline Verrucano & 0.0309 & 0.0309 & 0.01 & 0.03 & $1 \mathrm{E}-7$ \\
\hline Flysch & 0.15 & 0.15 & 0.15 & 0.06 & $1 \mathrm{E}-6$ \\
\hline LK & 6 & 0.5 & 0.5 & 0.05 & $1 \mathrm{E}-4$ \\
\hline Foot. carb. & 0.01 & 0.01 & 0.005 & 0.03 & $1 \mathrm{E}-7$ \\
\hline Hang. carb & 0.03091 & 0.03091 & 0.0309 & 0.04 & $1 \mathrm{E}-6$ \\
\hline
\end{tabular}
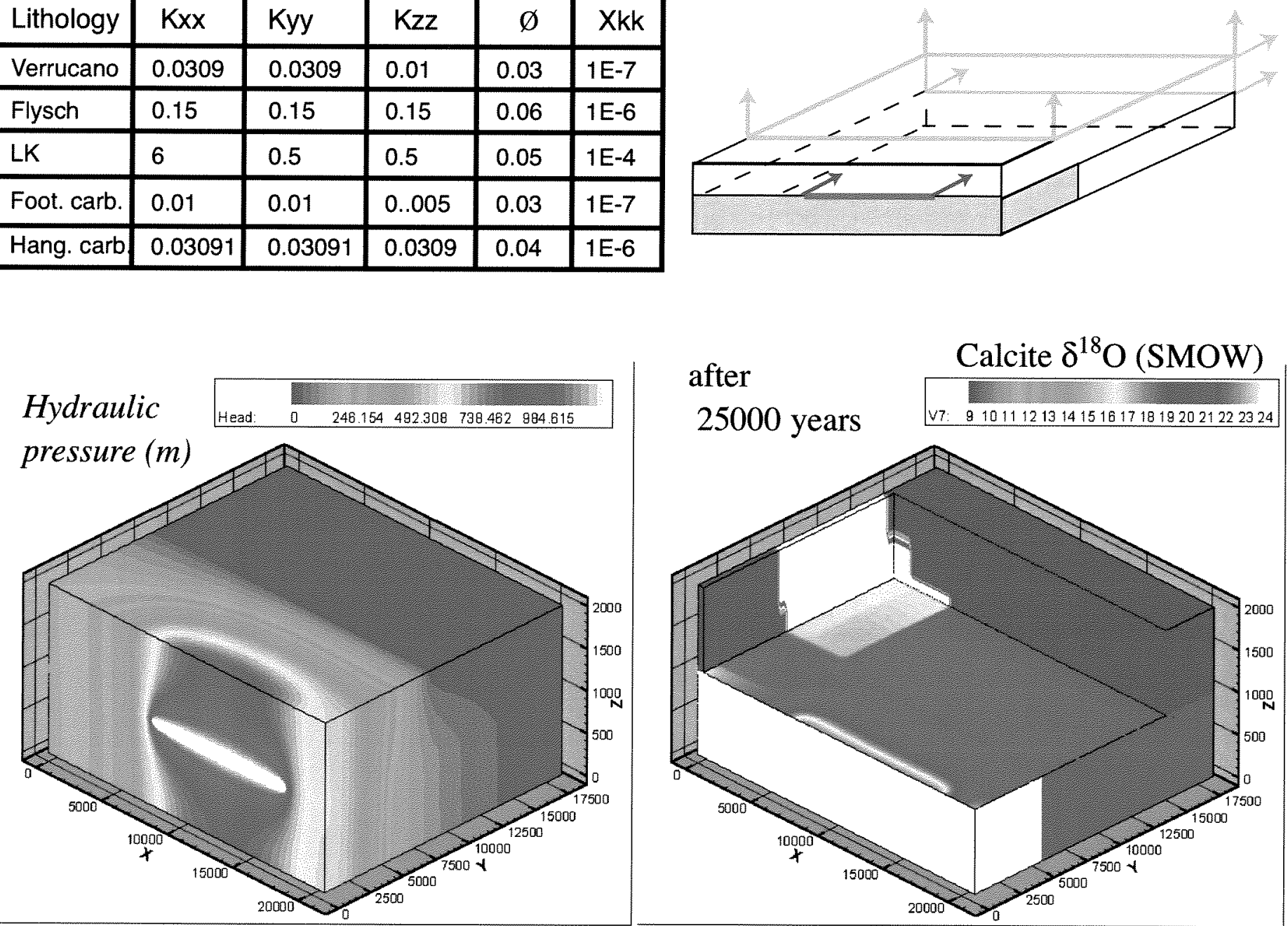

after

100000 years vi: 9101112131415161718192021222324

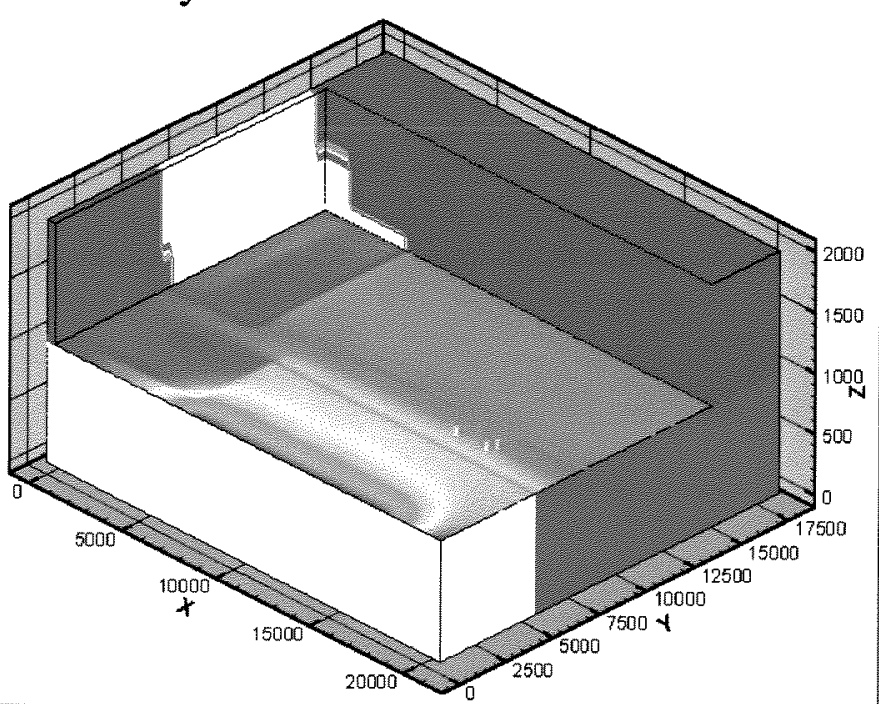

after

Calcite $\delta^{18} \mathrm{O}$ (SMOW)

500000 years 47.8101112131415181718192021222324

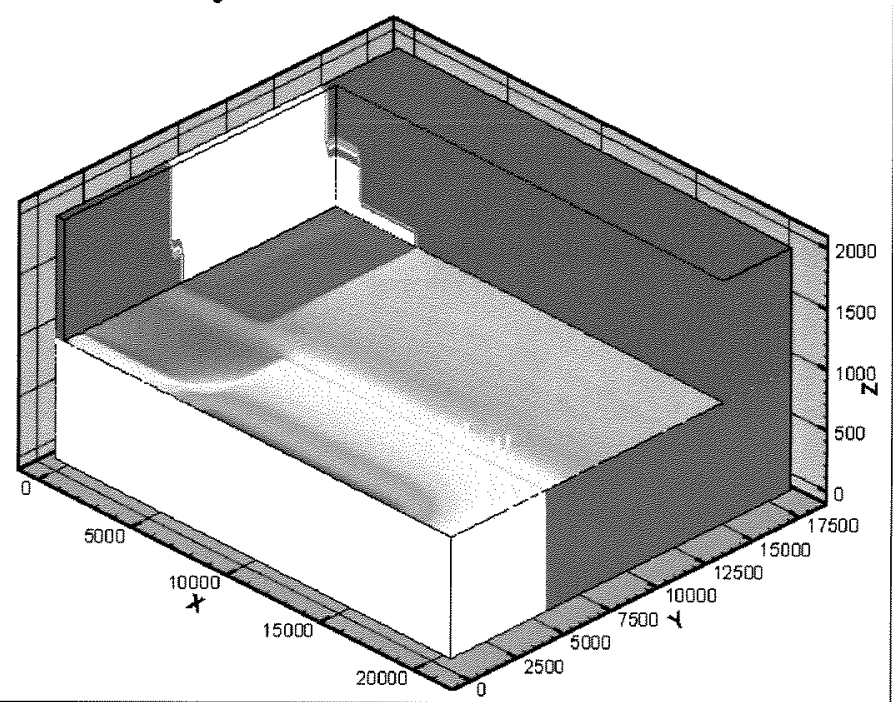




Basement head: 1200
\begin{tabular}{|l|l|l|c|c|c|}
\hline Lithology & Kxx & Kyy & Kzz & $\varnothing$ & Xkk \\
\hline Verrucano & 0.0309 & 0.0309 & 0.01 & 0.03 & $1 \mathrm{E}-7$ \\
\hline Flysch & 0.15 & 0.15 & 0.15 & 0.06 & $1 \mathrm{E}-6$ \\
\hline LK & 6 & 0.5 & 0.5 & 0.05 & $1 \mathrm{E}-4$ \\
\hline Foot. carb. & 0.01 & 0.01 & 0.005 & 0.03 & $1 \mathrm{E}-7$ \\
\hline Hang. carb & 0.03091 & 0.03091 & 0.0309 & 0.04 & $1 \mathrm{E}-6$ \\
\hline
\end{tabular}
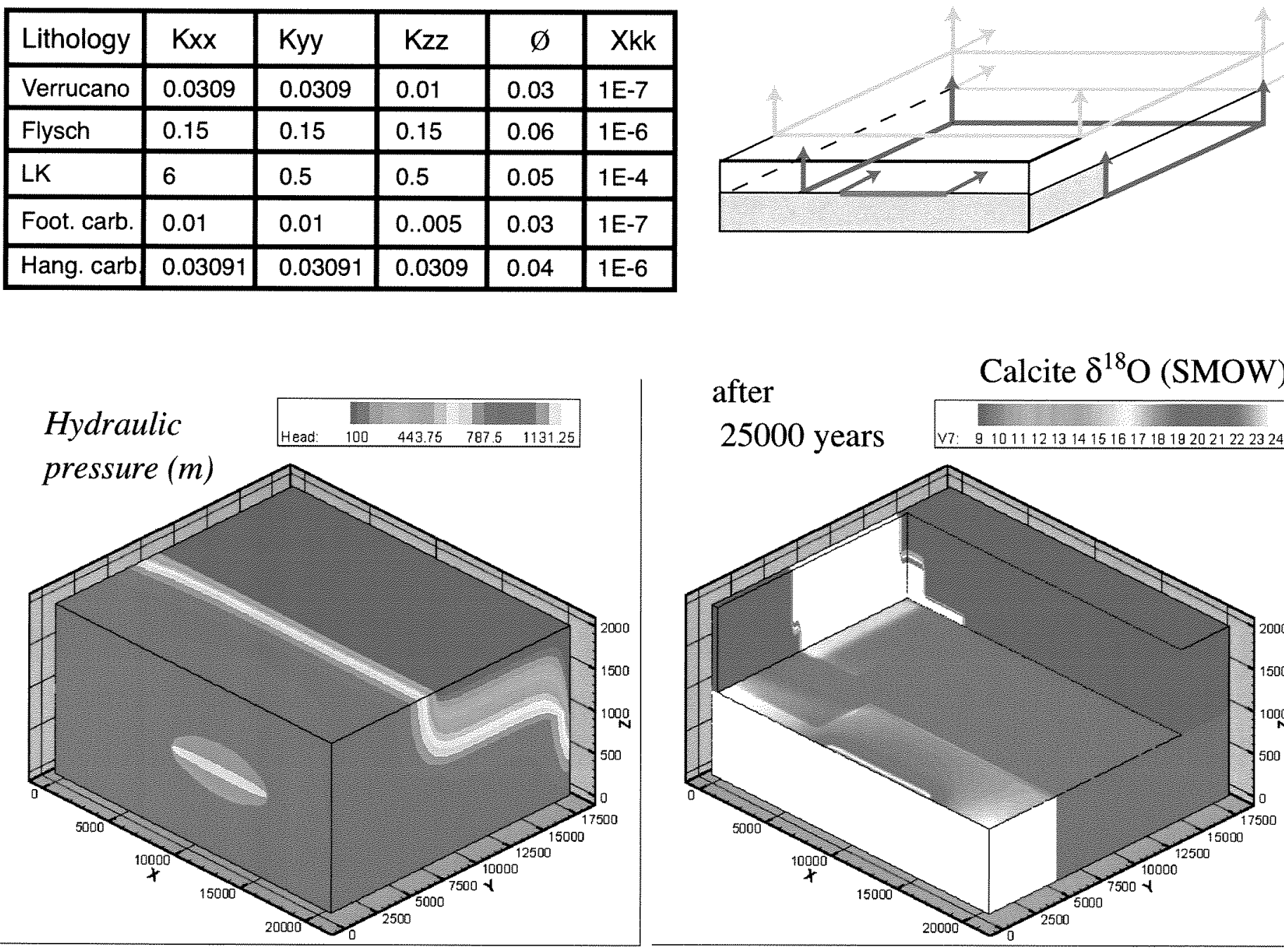

after

Calcite $\delta^{18} \mathrm{O}$ (SMOW)
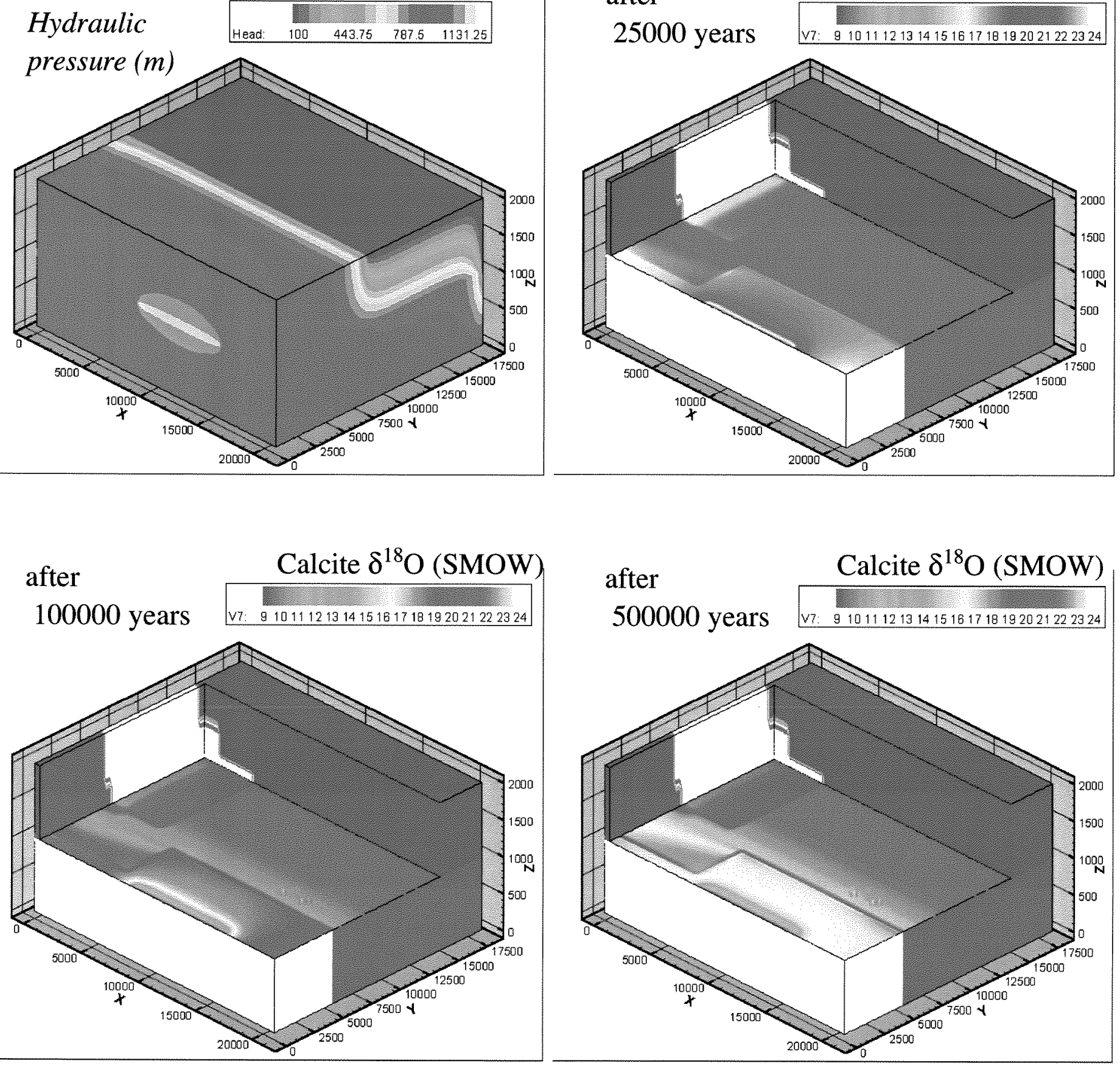


\section{S25}

Basement head: 1500

Flysch head: 900

\begin{tabular}{|l|l|l|l|l|l|}
\hline Lithology & $\mathrm{Kxx}$ & $\mathrm{Kyy}$ & $\mathrm{Kzz}$ & $\varnothing$ & Xkk \\
\hline Verrucano & 0.0309 & 0.0309 & 0.01 & 0.03 & $1 \mathrm{E}-6$ \\
\hline Flysch & 0.15 & 0.15 & 0.15 & 0.06 & $1 \mathrm{E}-6$ \\
\hline LK & 6 & 0.5 & 0.5 & 0.05 & $1 \mathrm{E}-4$ \\
\hline Foot. carb. & 0.01 & 0.01 & 0.005 & 0.03 & $1 \mathrm{E}-7$ \\
\hline Hang. carb & 0.03091 & 0.03091 & 0.0309 & 0.04 & $1 \mathrm{E}-6$ \\
\hline
\end{tabular}
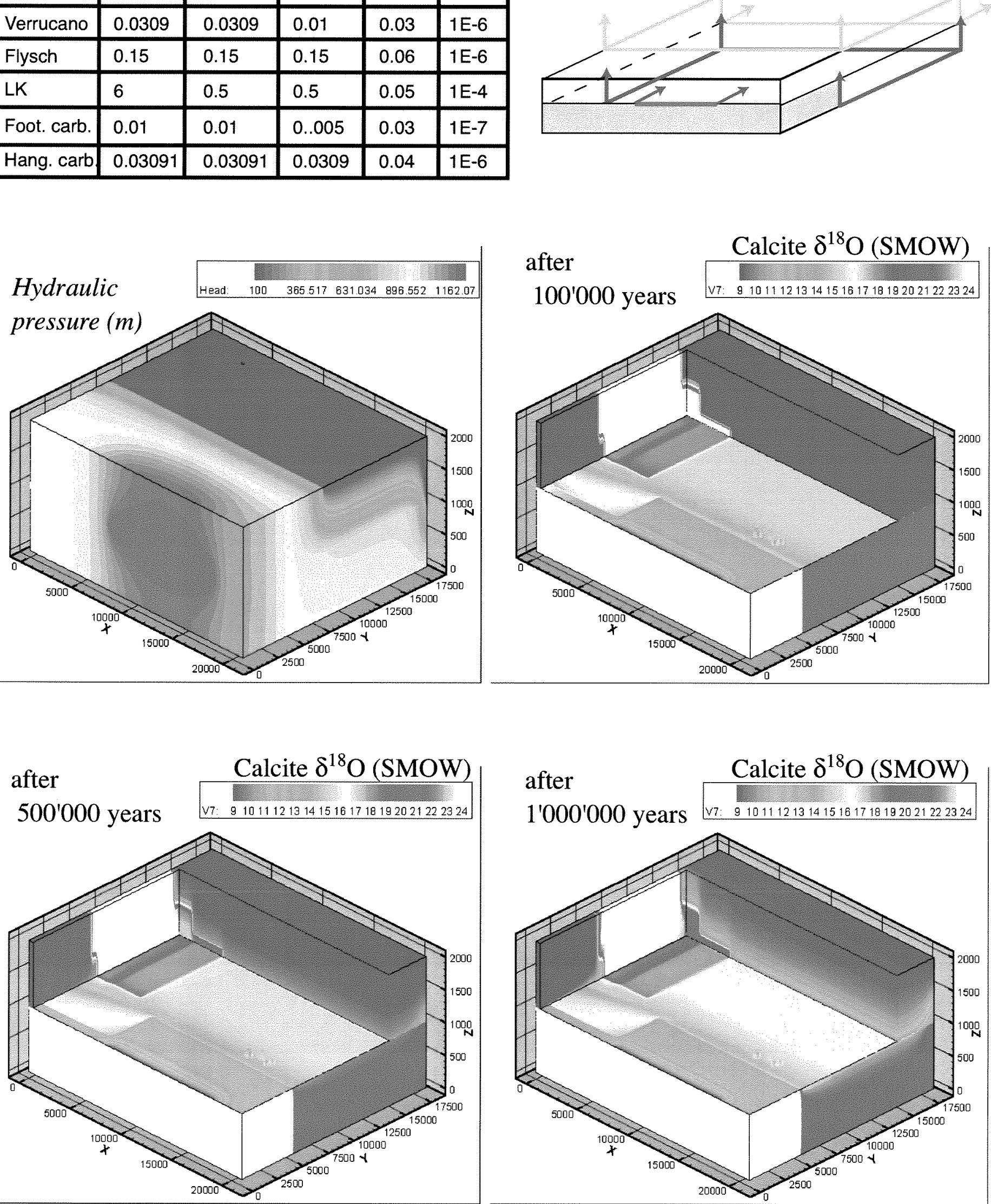
Basement head: 500

Flysch head: 500

\begin{tabular}{|l|l|l|l|l|l|}
\hline Lithology & Kxx & Kyy & Kzz & $\varnothing$ & Xkk \\
\hline Verrucano & 0.0309 & 0.0309 & 0.01 & 0.03 & $1 \mathrm{E}-7$ \\
\hline Flysch & 0.15 & 0.15 & 0.15 & 0.06 & $1 \mathrm{E}-6$ \\
\hline LK & 5 & 2 & 0.5 & 0.05 & $1 \mathrm{E}-4$ \\
\hline Foot. carb. & 0.02 & 0.02 & 0.005 & 0.03 & $1 \mathrm{E}-7$ \\
\hline Hang. carb & 0.03091 & 0.03091 & 0.0309 & 0.04 & $1 \mathrm{E}-6$ \\
\hline
\end{tabular}
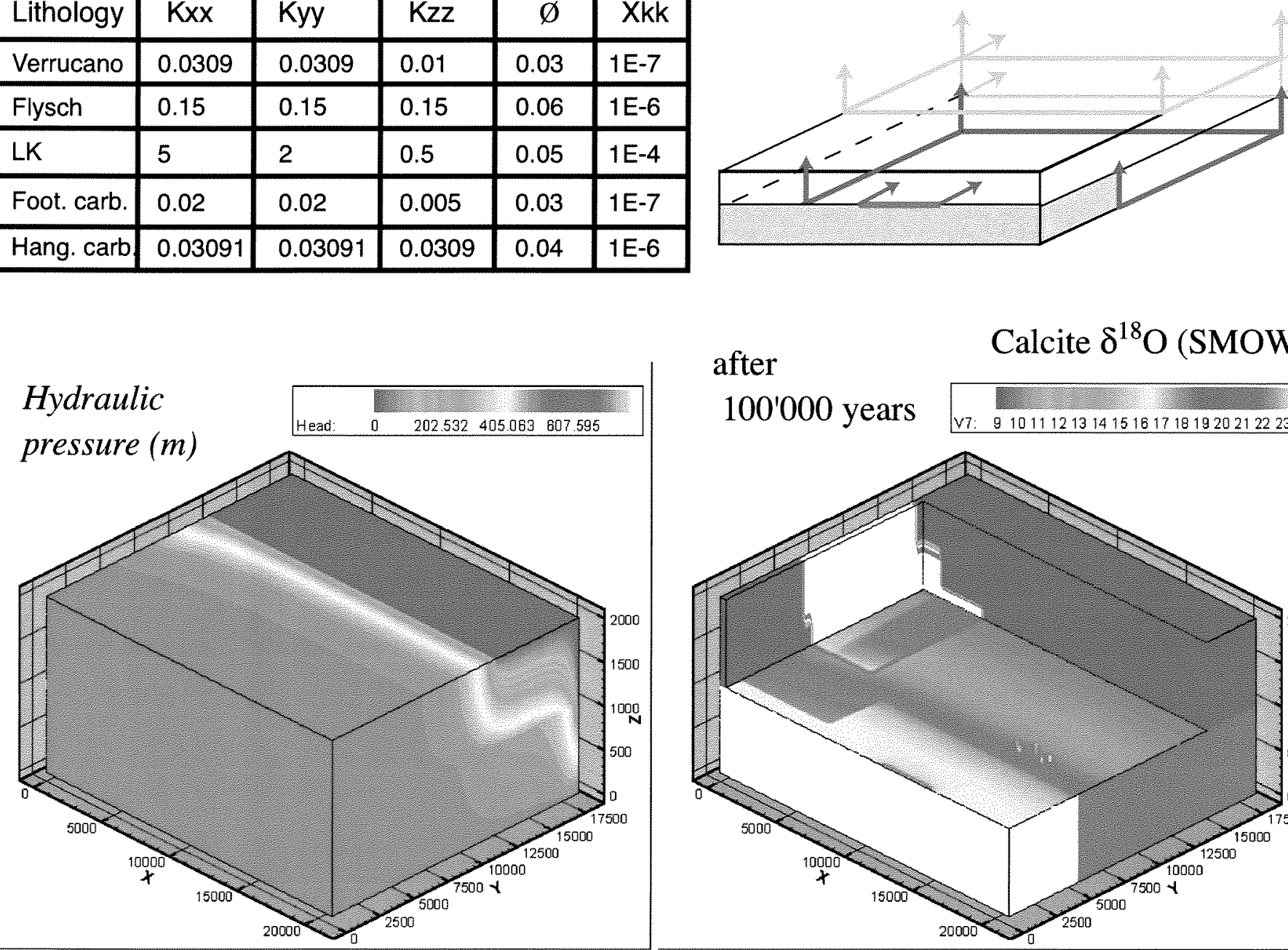

after

Calcite $\delta^{18} \mathrm{O}$ (SMOW)

100'000 years v7: 9101112131415161718192021222324
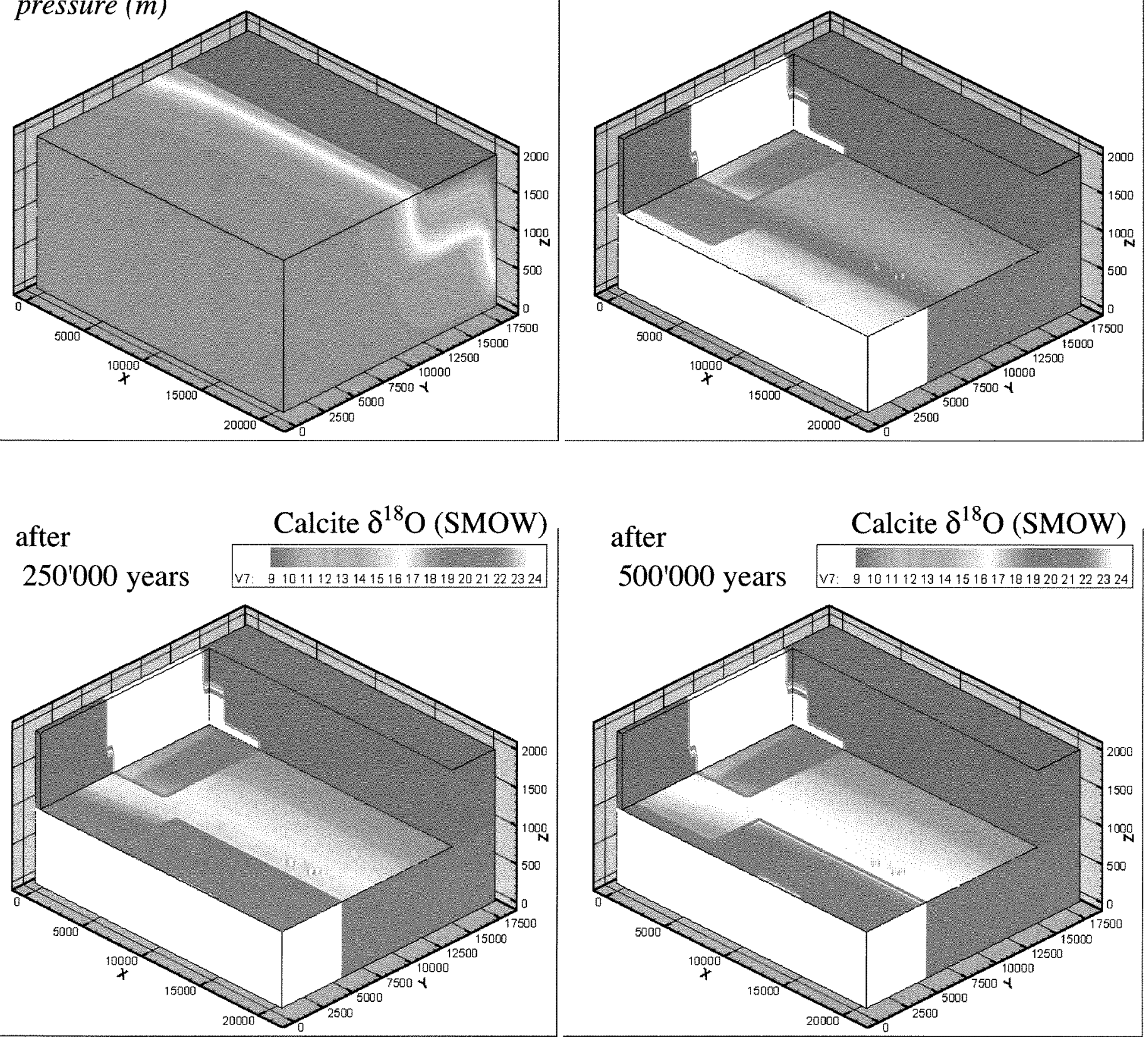


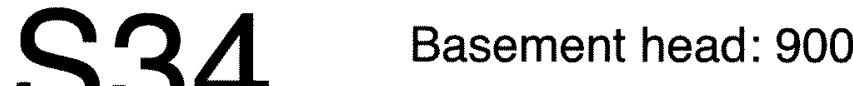 \\ Flysch head: 500}

\begin{tabular}{|l|l|l|l|l|l|}
\hline Lithology & $\mathrm{Kxx}$ & Kyy & $\mathrm{Kzz}$ & $\varnothing$ & Xkk \\
\hline Verrucano & 0.0309 & 0.0309 & 0.01 & 0.03 & $1 \mathrm{E}-7$ \\
\hline Flysch & 0.15 & 0.15 & 0.15 & 0.06 & $1 \mathrm{E}-6$ \\
\hline LK & 4 & 0.5 & 0.5 & 0.08 & $1 \mathrm{E}-4$ \\
\hline Foot. carb. & 0.02 & 0.02 & 0.005 & 0.03 & $1 \mathrm{E}-7$ \\
\hline Hang. carb & 0.03091 & 0.03091 & 0.0309 & 0.04 & $1 \mathrm{E}-6$ \\
\hline
\end{tabular}
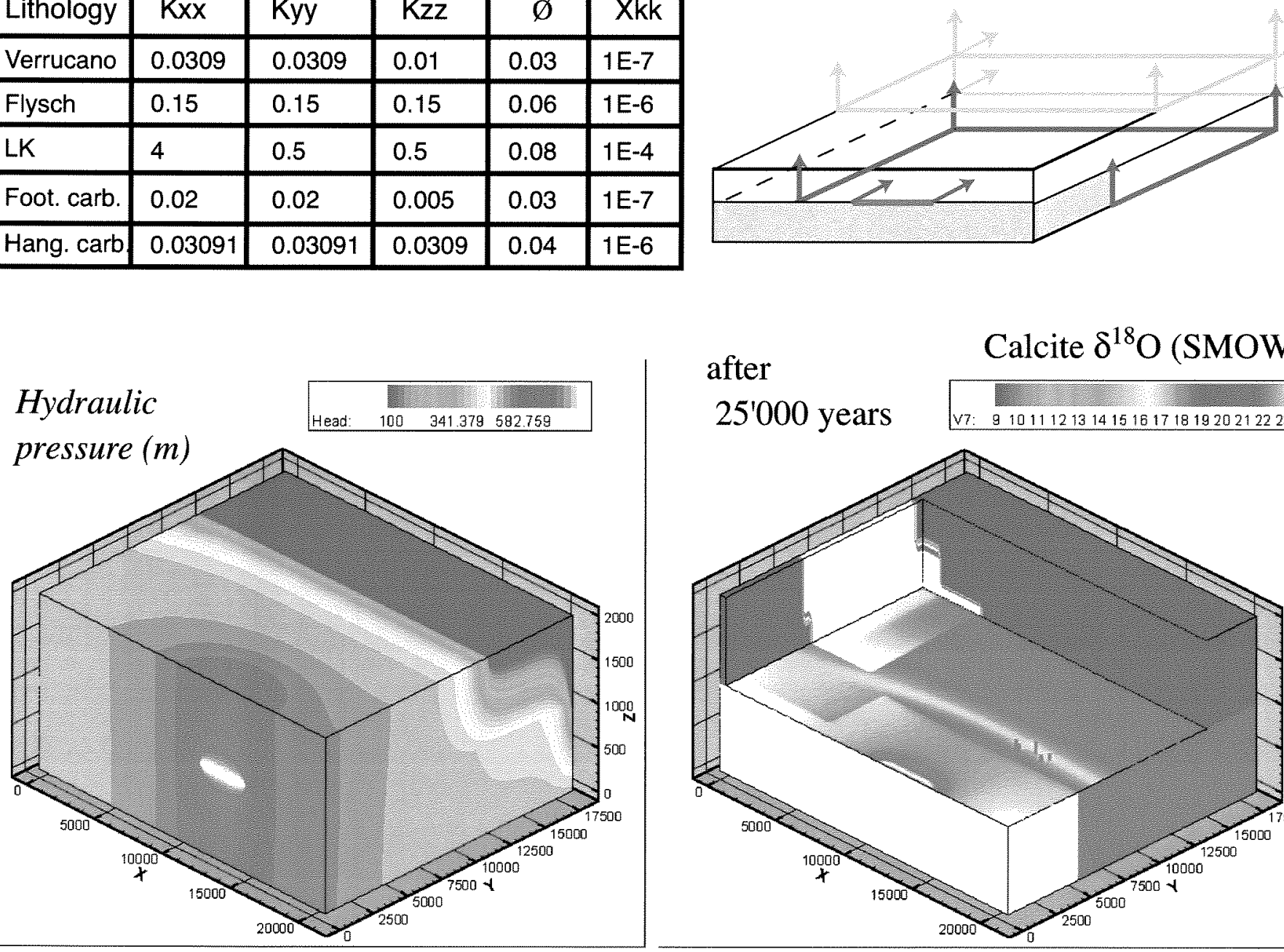

after

Calcite $\delta^{18} \mathrm{O}$ (SMOW)
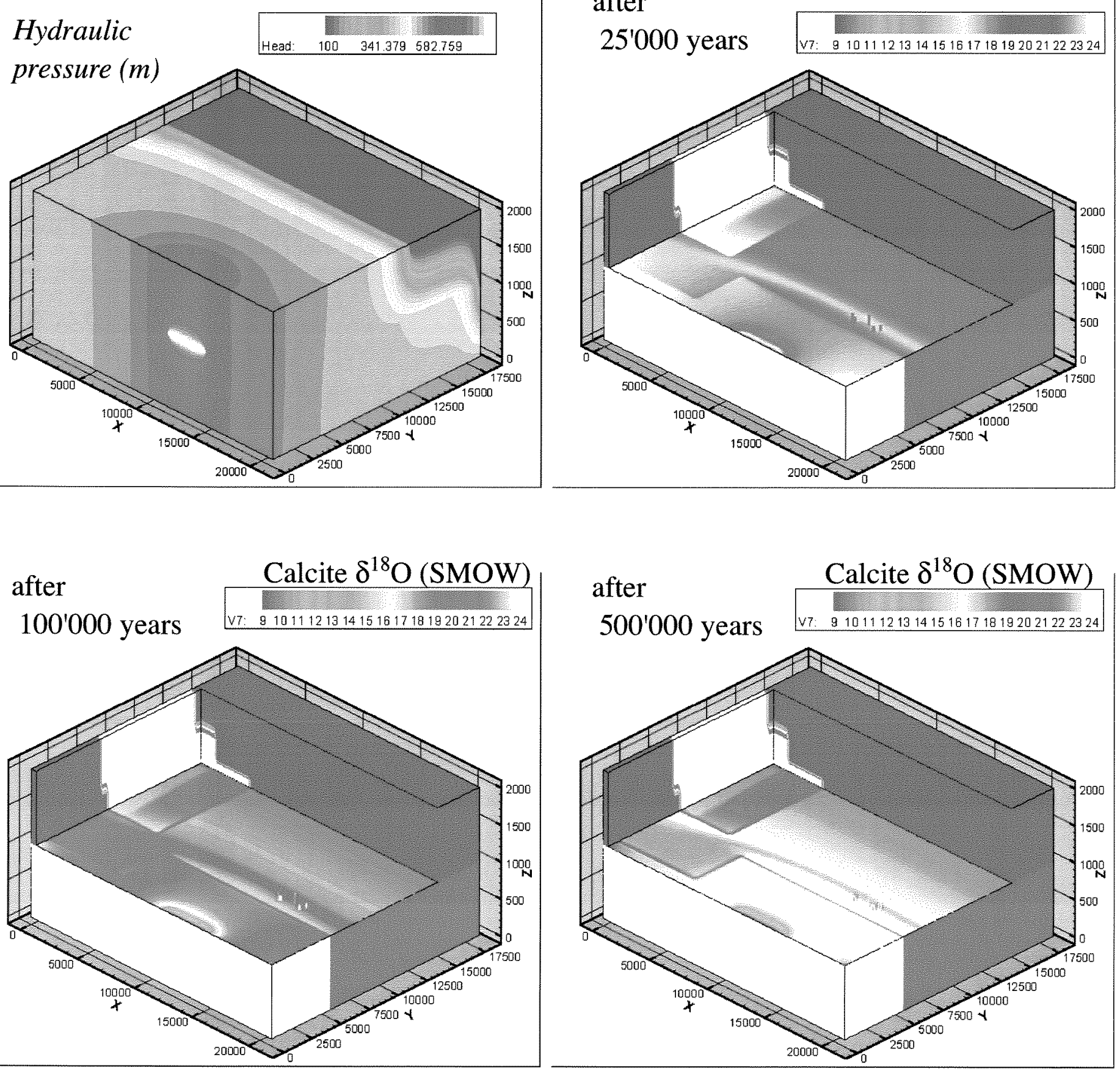

after

Calcite $\delta^{18} \mathrm{O}$ (SMOW)

500'000 years vi: 9101112131415161718192021222324

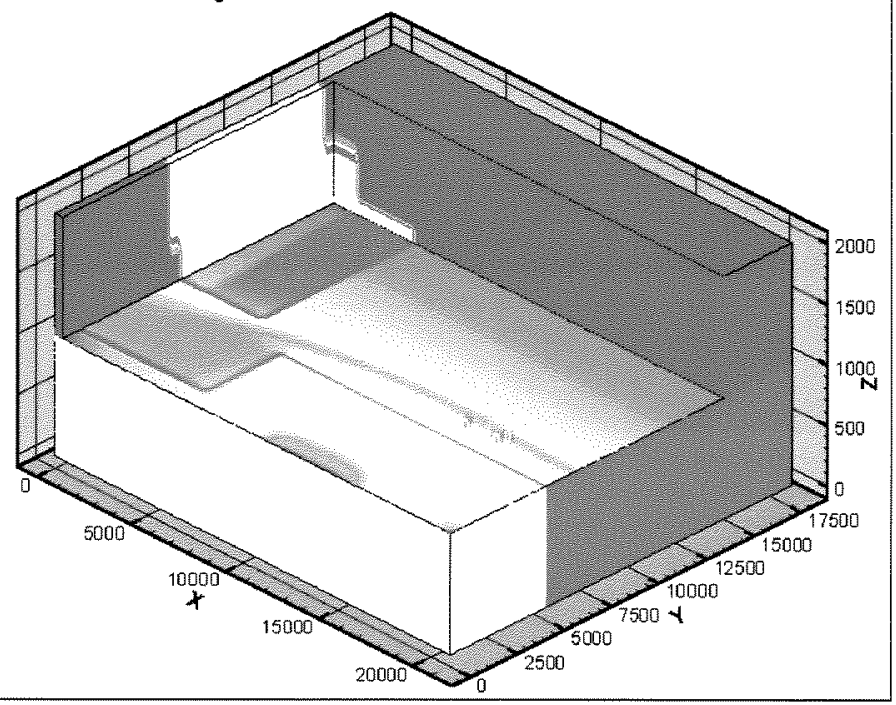




\begin{tabular}{|l|l|l|l|l|l|}
\hline Lithology & $\mathrm{Kxx}$ & $\mathrm{Kyy}$ & $\mathrm{Kzz}$ & $\varnothing$ & $\mathrm{Xkk}$ \\
\hline Verrucano & 0.0309 & 0.0309 & 0.01 & 0.03 & $1 \mathrm{E}-7$ \\
\hline Flysch & 0.15 & 0.15 & 0.15 & 0.06 & $1 \mathrm{E}-6$ \\
\hline LK & 6 & 0.5 & 0.5 & 0.05 & $1 \mathrm{E}-4$ \\
\hline Foot. carb. & 0.01 & 0.01 & 0.005 & 0.03 & $1 \mathrm{E}-7$ \\
\hline Hang. carb & 0.03091 & 0.03091 & 0.0309 & 0.04 & $1 \mathrm{E}-6$ \\
\hline
\end{tabular}
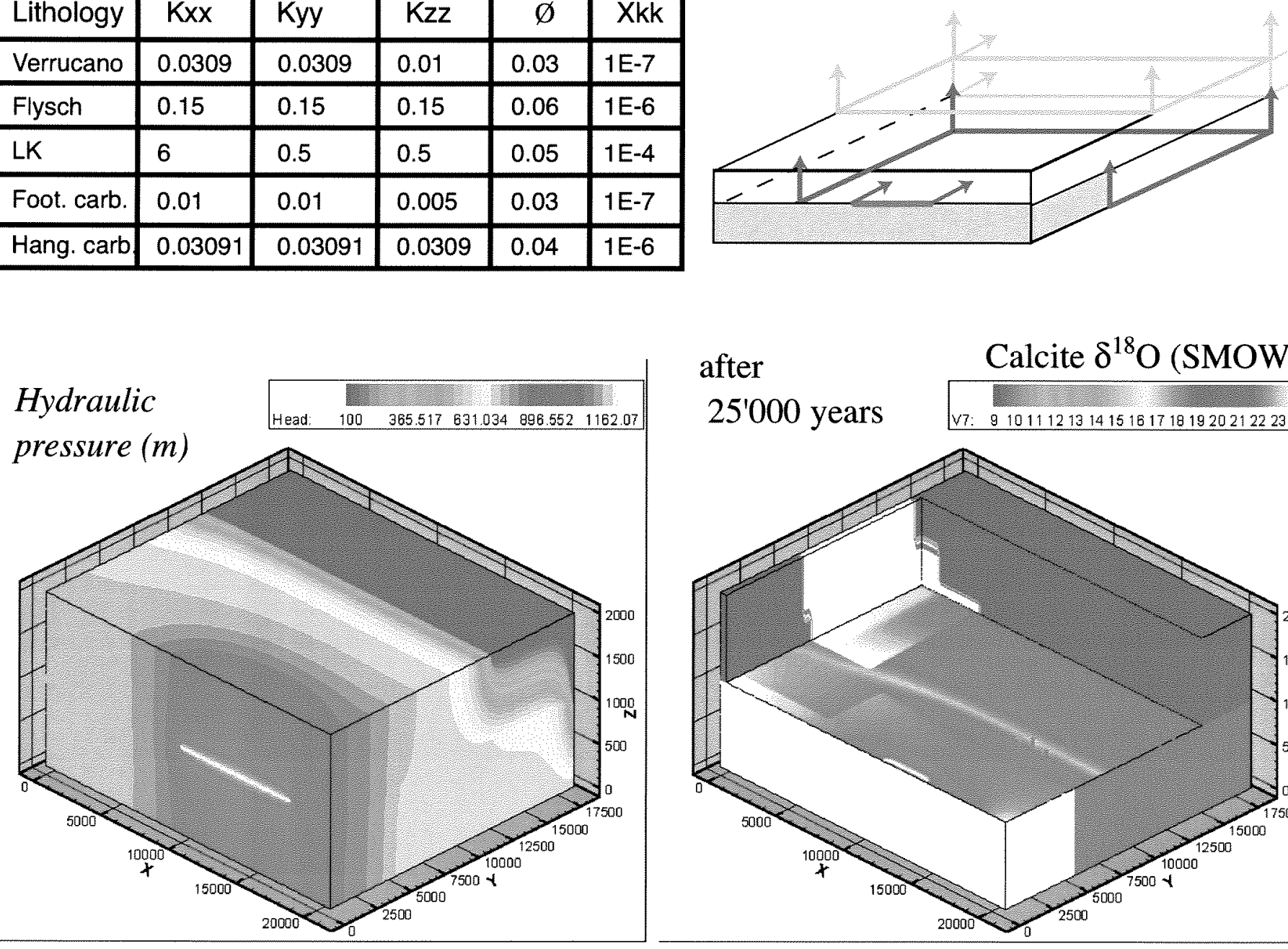

after

Calcite $\delta^{18} \mathrm{O}$ (SMOW)

25'000 years $\quad$ 7: 9101112131415151719192021222324
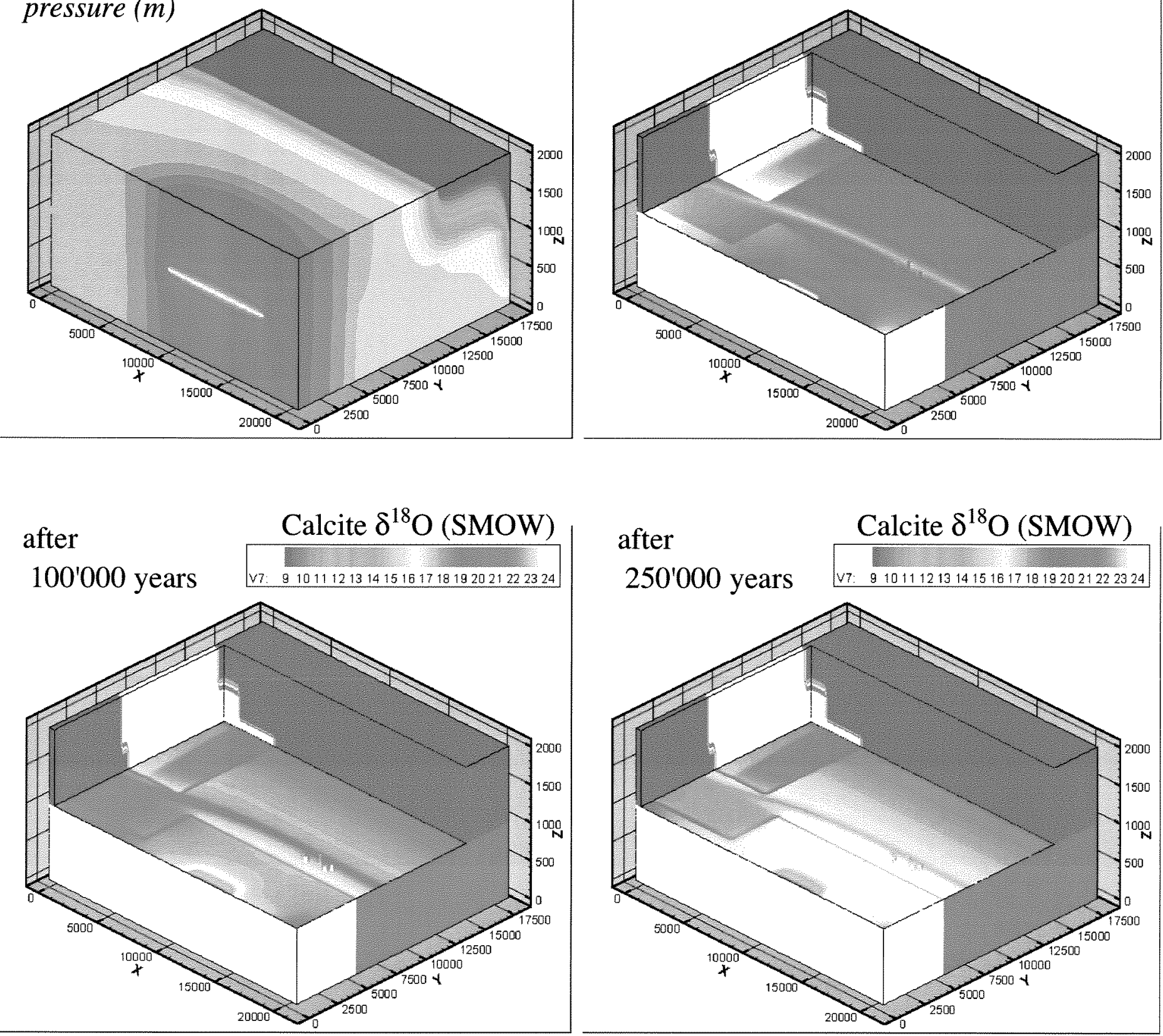


 \\ Flysch head: 500}

\begin{tabular}{|l|l|l|l|l|l|}
\hline Lithology & $\mathrm{Kxx}$ & Kyy & $\mathrm{Kzz}$ & $\varnothing$ & Xkk \\
\hline Verrucano & 0.0309 & 0.0309 & 0.01 & 0.03 & $1 \mathrm{E}-7$ \\
\hline Flysch & 0.15 & 0.15 & 0.15 & 0.06 & $1 \mathrm{E}-6$ \\
\hline LK & 2 & 2 & 2 & 0.05 & $1 \mathrm{E}-4$ \\
\hline Foot. carb. & 0.02 & 0.02 & 0.005 & 0.03 & $1 \mathrm{E}-7$ \\
\hline Hang. carb & 0.03091 & 0.03091 & 0.0309 & 0.04 & $1 \mathrm{E}-6$ \\
\hline
\end{tabular}



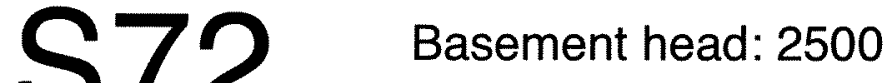 \\ Flysch head: 900}

\begin{tabular}{|l|l|l|l|l|l|}
\hline Lithology & Kxx & Kyy & Kzz & $\varnothing$ & Xkk \\
\hline Verrucano & 0.0309 & 0.0309 & 0.01 & 0.03 & $1 \mathrm{E}-7$ \\
\hline Flysch & 0.15 & 0.15 & 0.15 & 0.06 & $1 \mathrm{E}-6$ \\
\hline LK & 6 & 0.5 & 0.5 & 0.05 & $1 \mathrm{E}-4$ \\
\hline Foot. carb. & 0.01 & 0.01 & 0.005 & 0.03 & $1 \mathrm{E}-7$ \\
\hline Hang. carb & 0.03091 & 0.03091 & 0.0309 & 0.04 & $1 \mathrm{E}-6$ \\
\hline
\end{tabular}



\section{S76 Basenent traat: 1500 \\ Flysch head: 900}

\begin{tabular}{|l|l|l|l|l|l|}
\hline Lithology & $\mathrm{Kxx}$ & $\mathrm{Kyy}$ & $\mathrm{Kzz}$ & $\varnothing$ & Xkk \\
\hline Verrucano & 0.0309 & 0.0309 & 0.01 & 0.03 & $1 \mathrm{E}-7$ \\
\hline Flysch & 0.15 & 0.15 & 0.15 & 0.06 & $1 \mathrm{E}-6$ \\
\hline LK & 6 & 0.5 & 0.5 & 0.05 & $1 \mathrm{E}-4$ \\
\hline Foot. carb. & 0.01 & 0.01 & 0.005 & 0.03 & $1 \mathrm{E}-7$ \\
\hline Hang. carb & 0.03091 & 0.03091 & 0.0309 & 0.04 & $1 \mathrm{E}-6$ \\
\hline
\end{tabular}
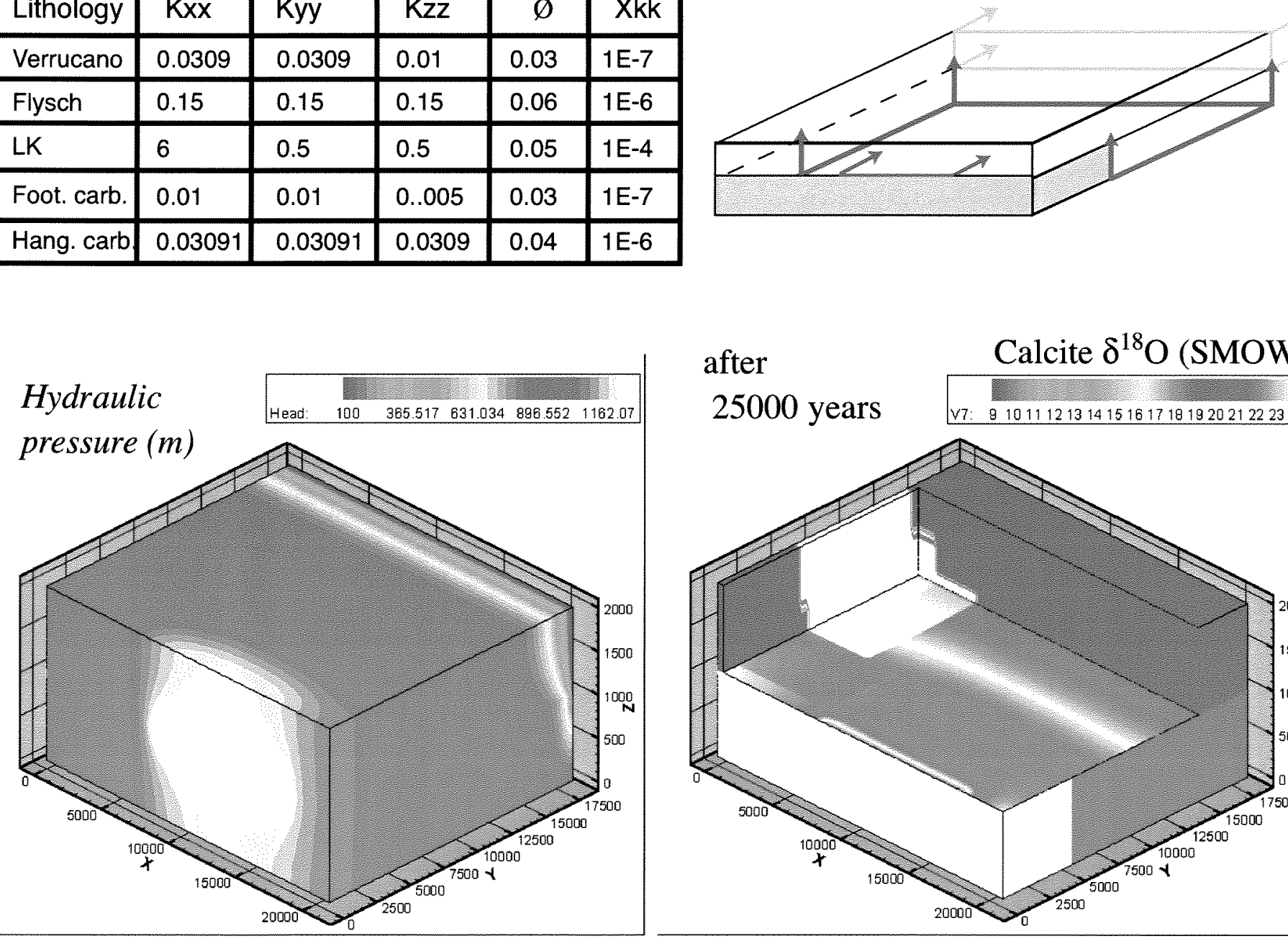

after

Calcite $\delta^{18} \mathrm{O}$ (SMOW)
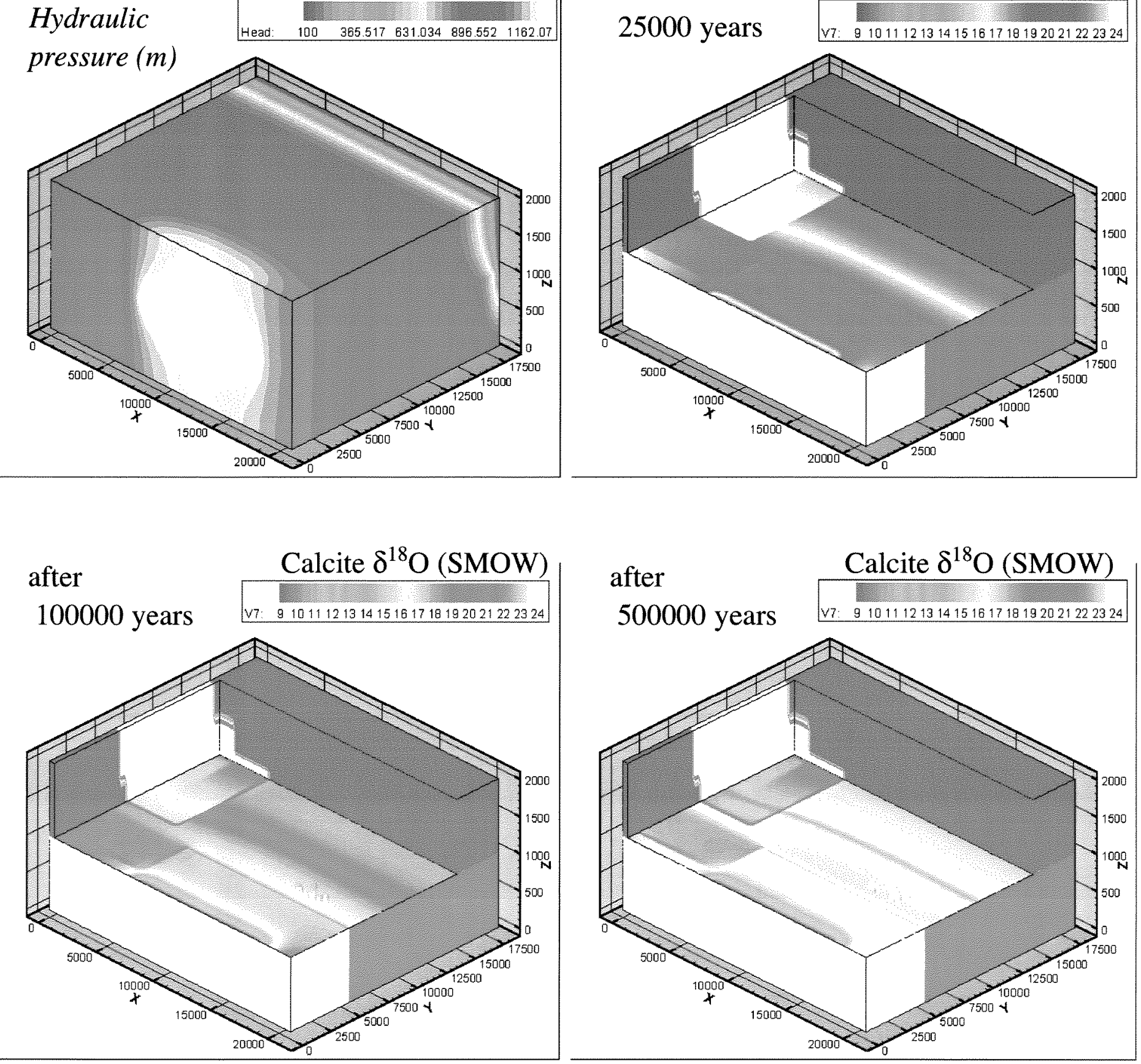


\begin{tabular}{|l|l|l|l|l|l|}
\hline Lithology & $\mathrm{Kxx}$ & $\mathrm{Kyy}$ & $\mathrm{Kzz}$ & $\varnothing$ & $\mathrm{Xkk}$ \\
\hline Verrucano & 0.0309 & 0.0309 & 0.01 & 0.03 & $1 \mathrm{E}-7$ \\
\hline Flysch & 0.15 & 0.15 & 0.15 & 0.06 & $1 \mathrm{E}-6$ \\
\hline LK & 6 & 0.5 & 0.5 & 0.05 & $1 \mathrm{E}-4$ \\
\hline Foot. carb. & 0.01 & 0.01 & $0 . .005$ & 0.03 & $1 \mathrm{E}-7$ \\
\hline Hang. carb & 0.03091 & 0.03091 & 0.0309 & 0.04 & $1 \mathrm{E}-6$ \\
\hline
\end{tabular}


after

Calcite $\delta^{18} \mathrm{O}$ (SMOW)

100'000 years v7: 9101112131415161718192021222324
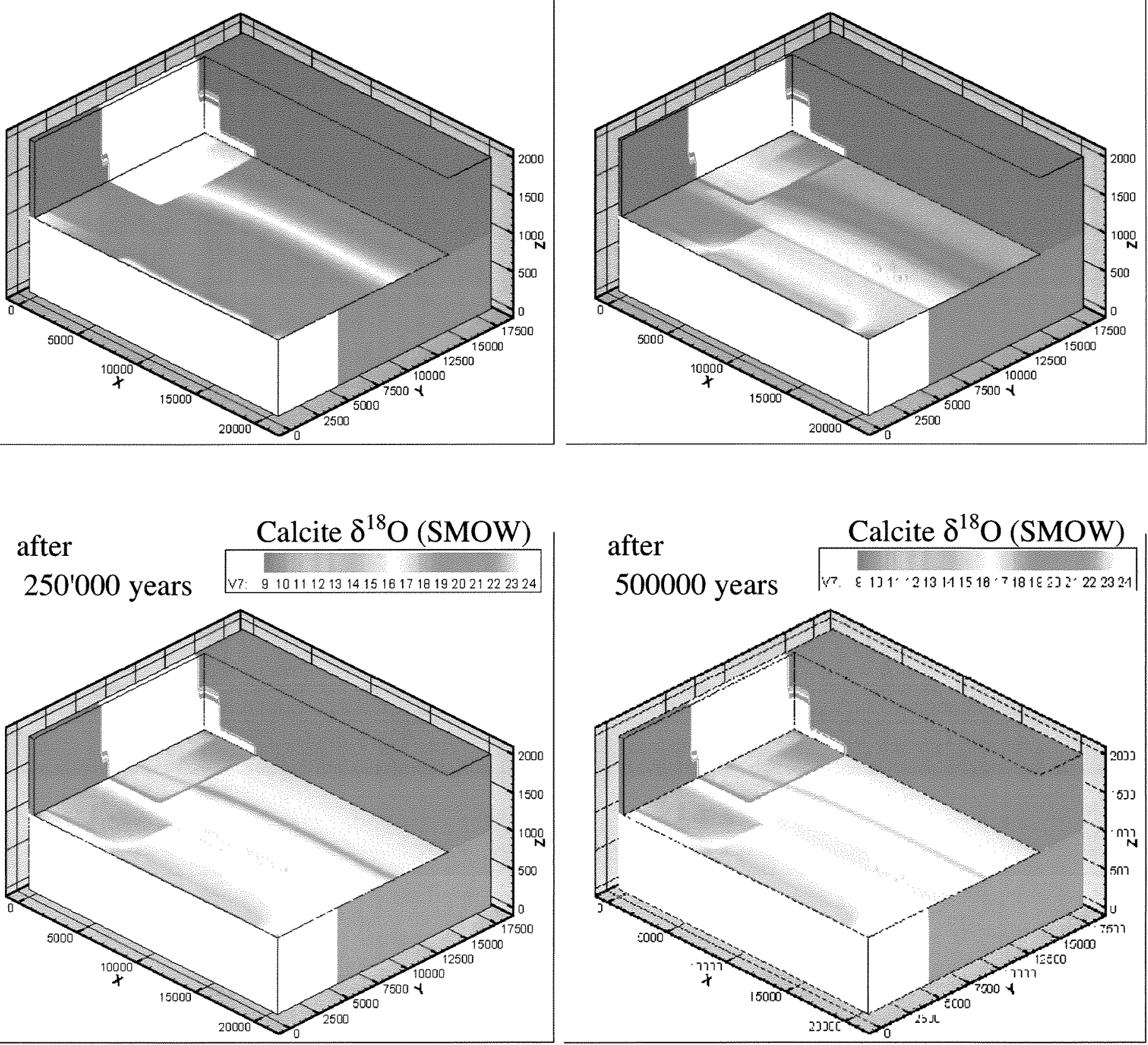

after

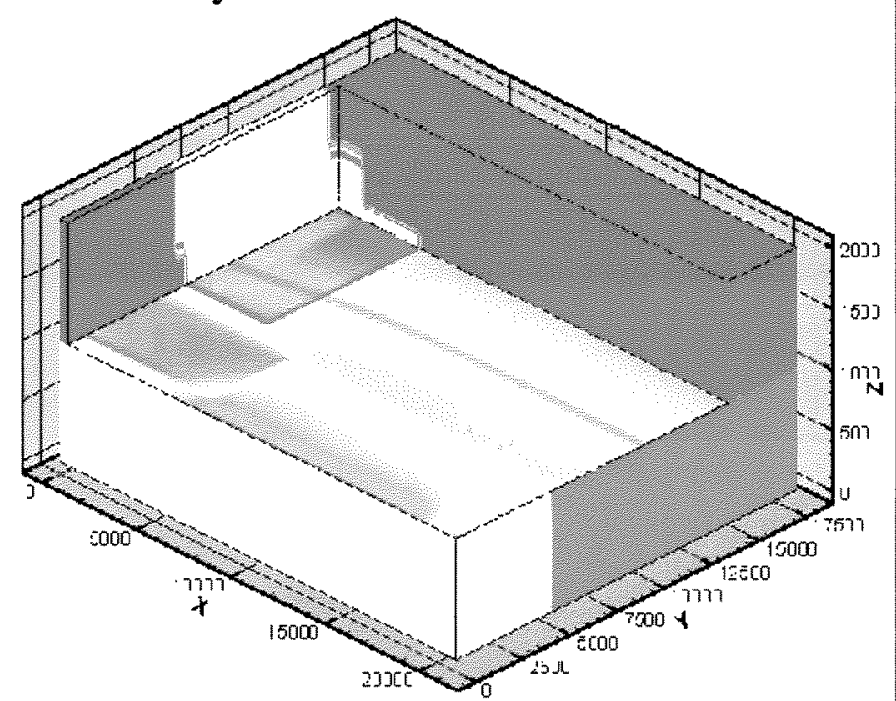




\section{S78 Basernat rasat 1500 \\ Flysch head: 900}

\begin{tabular}{|l|l|l|l|l|l|}
\hline Lithology & $\mathrm{Kxx}$ & $\mathrm{Kyy}$ & $\mathrm{Kzz}$ & $\varnothing$ & $\mathrm{Xkk}$ \\
\hline Verrucano & 0.0309 & 0.0309 & 0.01 & 0.03 & $1 \mathrm{E}-7$ \\
\hline Flysch & 0.15 & 0.15 & 0.15 & 0.06 & $1 \mathrm{E}-6$ \\
\hline LK & 6 & 0.5 & 0.5 & 0.05 & $1 \mathrm{E}-4$ \\
\hline Foot. carb. & 0.01 & 0.01 & 0.005 & 0.03 & $1 \mathrm{E}-7$ \\
\hline Hang. carb & 0.03091 & 0.03091 & 0.0309 & 0.04 & $1 \mathrm{E}-6$ \\
\hline
\end{tabular}
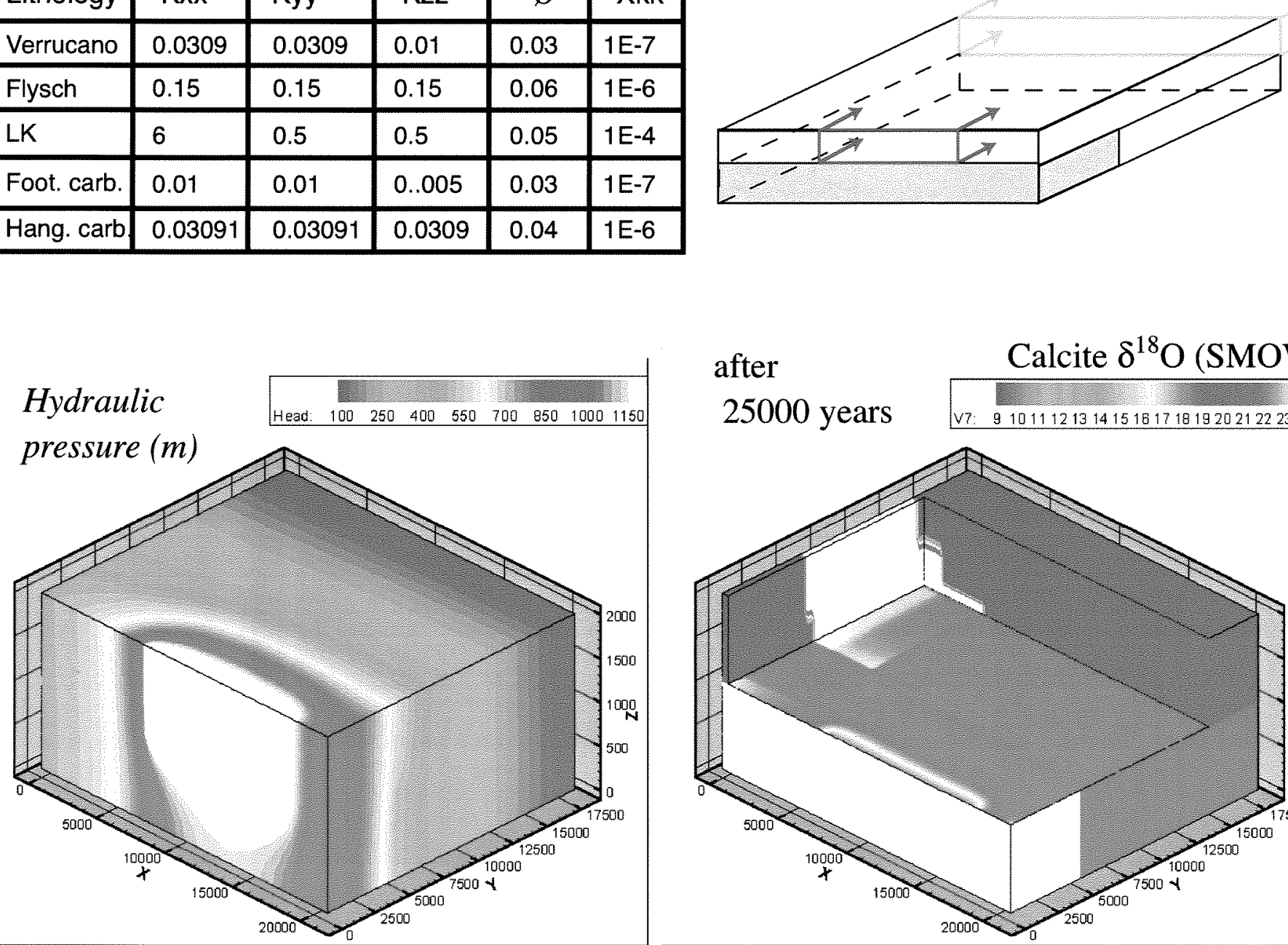

after

Calcite $\delta^{18} \mathrm{O}$ (SMOW) 25000 years
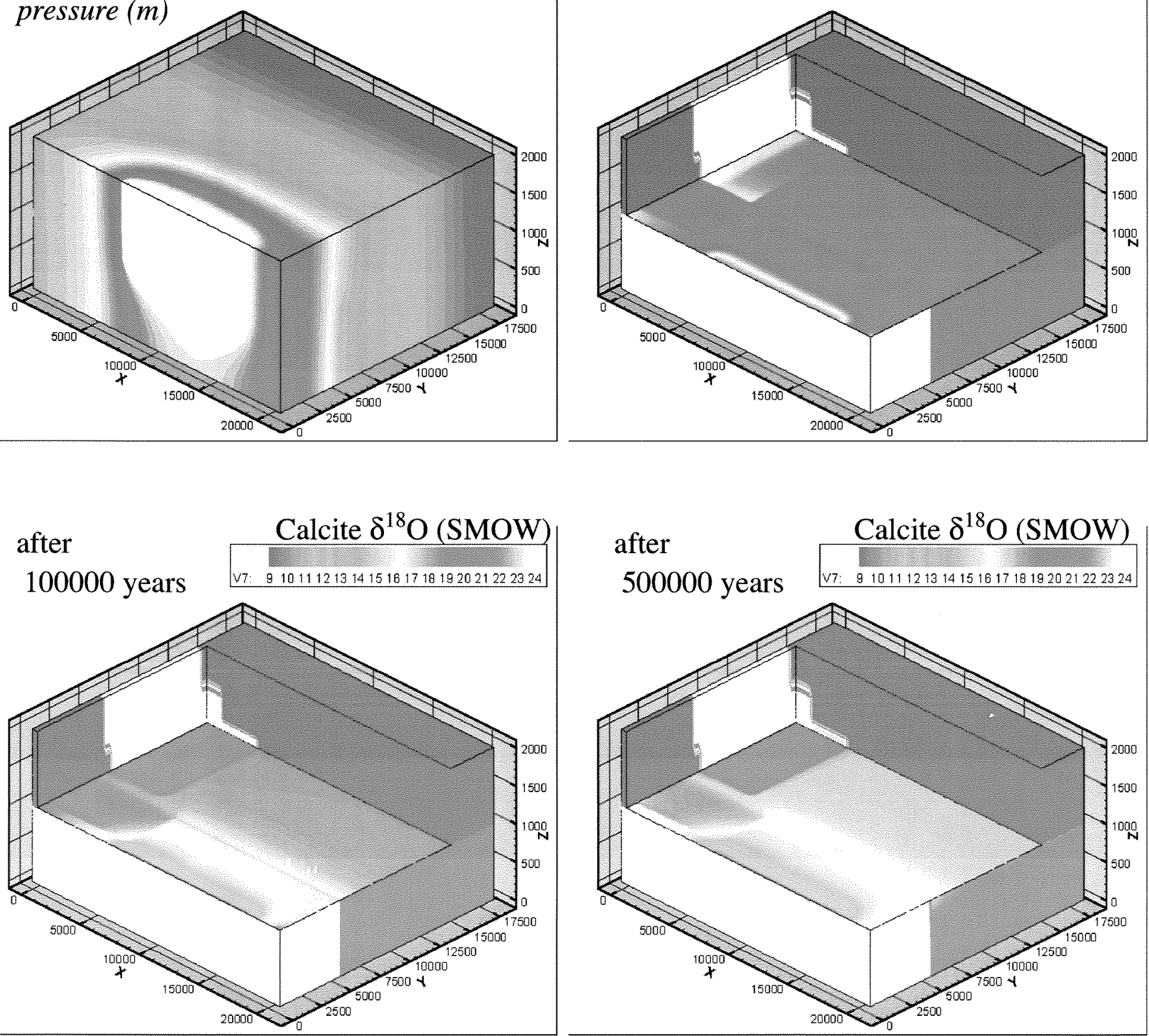


\begin{tabular}{|l|l|l|l|l|l|}
\hline Lithology & $\mathrm{Kxx}$ & $\mathrm{Kyy}$ & $\mathrm{Kzz}$ & $\varnothing$ & $\mathrm{Xkk}$ \\
\hline Verrucano & 0.0309 & 0.0309 & 0.01 & 0.03 & $1 \mathrm{E}-7$ \\
\hline Flysch & 0.15 & 0.15 & 0.15 & 0.06 & $1 \mathrm{E}-6$ \\
\hline LK & 6 & 0.5 & 0.5 & 0.05 & $1 \mathrm{E}-4$ \\
\hline Foot. carb. & 0.01 & 0.01 & 0.005 & 0.03 & $1 \mathrm{E}-7$ \\
\hline Hang. carb & 0.03091 & 0.03091 & 0.0309 & 0.04 & $1 \mathrm{E}-6$ \\
\hline
\end{tabular}
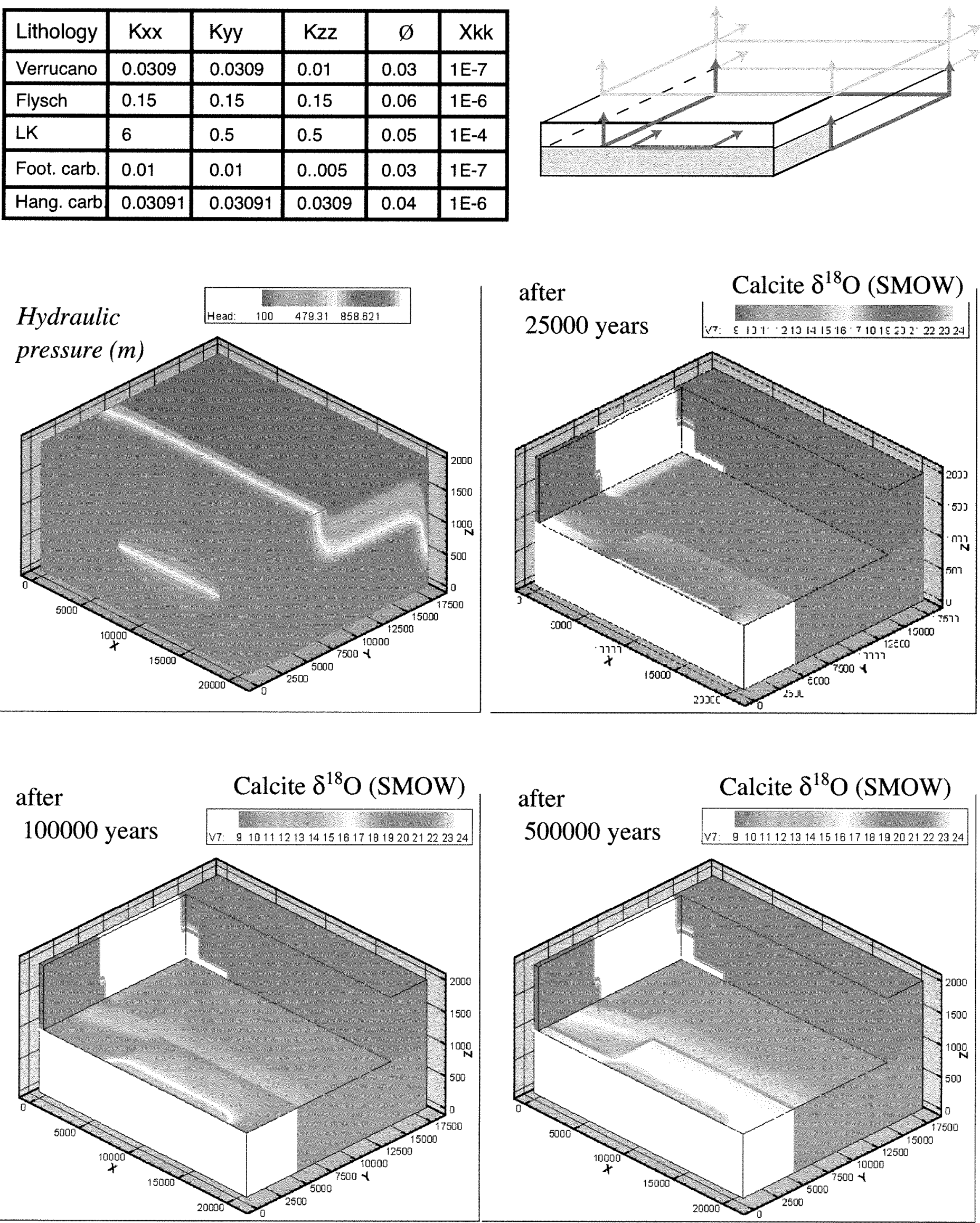